\title{
A characterisation result on a particular class of non-weighted minihypers
}

\author{
J. De Beule, A. Hallez and L. Storme ${ }^{\dagger}$
}

\begin{abstract}
We present a characterisation of $\left\{\epsilon_{1}(q+1)+\epsilon_{0}, \epsilon_{1} ; n, q\right\}$-minihypers, $q$ square, $q=p^{h}, p>3$ prime, $h \geq 2, q \geq 1217$, for $\epsilon_{0}+\epsilon_{1}<\frac{q^{7 / 12}}{2}-\frac{q^{1 / 4}}{2}$. This improves a characterisation result of S. Ferret and L. Storme [6], involving more Baer subgeometries contained in the minihyper.
\end{abstract}

Keywords: minihypers, Griesmer bound, blocking sets

\section{Introduction}

Let $\mathrm{PG}(n, q)$ be the $n$-dimensional projective space over the finite field $\mathbb{F}_{q}$ of order $q$. A weight function $w$ of $\mathrm{PG}(n, q)$ is a mapping from the point set of $\mathrm{PG}(n, q)$ to the set of non-negative integers. For a point $P$, the integer $w(P)$ is called the weight of the point $P$, and for a set $M$ of points, its weight is the sum of the weights of its points. The sum of the weights of all points of $\operatorname{PG}(n, q)$ is the total weight of $w$.

Definition 1.1 An $\{f, m ; n, q\}$-minihyper, $f \geq 1, n \geq 2$, is a pair $(F, w)$, where $F$ is a set of points of $\mathrm{PG}(n, q), w$ is a weight function of $\mathrm{PG}(n, q)$, and

*This author is a postdoctoral research fellow of the Research Foundation Flanders Belgium (FWO).

${ }^{\dagger}$ This research was initiated while the third author was visiting the Justus-LiebigUniversität Gießen, Germany, with a Fellowship of the Alexander von Humboldt Foundation. 
$P \in F \Longleftrightarrow w(P)>0$; the total weight of $w$ is $f$, and $m$ is the minimum weight of the hyperplanes of $\mathrm{PG}(n, q)$, i.e.,

$$
\sum_{P \in \pi} w(P) \geq m
$$

for any hyperplane $\pi$ of $\mathrm{PG}(n, q)$, with equality for at least one hyperplane of $\mathrm{PG}(n, q)$.

Of course, the set $F$ is determined by the weight function $w$. When the range of $w$ is $\{0,1\}$, the converse is true and then the minihyper is identified with $F$ and is called a non-weighted minihyper. Thus, a nonweighted $\{f, m ; n, q\}$-minihyper is a set $F$ of $f$ points of $\operatorname{PG}(n, q)$ such that every hyperplane contains at least $m$ points of $F$. This is the definition of a minihyper given by Hamada and Tamari in [12] and it was generalised to the definition of a weighted minihyper in [7]. (Weighted) minihypers can also be called (weighted) multiple blocking sets. In the sequel, we will always clearly distinguish between weighted and non-weighted minihypers and use the term minihyper to denote a non-weighted minihyper.

As (weighted) minihypers are (weighted) multiple blocking sets with respect to the hyperplanes of a projective space, the study of these objects fits completely in the rich literature on the study of blocking sets and generalisations. But (weighted) minihypers are also a geometric interpretation of linear codes meeting the Griesmer bound. This connection is described in detail in several references, such as $[4,5]$.

Denote the number of points in an $i$-dimensional projective space $\operatorname{PG}(i, q)$ by $\theta_{i}$, i.e., $\theta_{i}=\frac{q^{i+1}-1}{q-1}$, and define $\theta_{-1}=0$. The following characterisation theorem was shown by Hamada, Helleseth and Maekawa.

Theorem $1.2([10,11]) A\left\{\sum_{i=0}^{s} \epsilon_{i} \theta_{i}, \sum_{i=0}^{s} \epsilon_{i} \theta_{i-1} ; n, q\right\}$-minihyper is the union of pairwise disjoint $\epsilon_{i}$ projective subspaces of dimension $i$, for $i=0, \ldots, s$, if $\sum_{i=0}^{s} \epsilon_{i}=h<\sqrt{q}+1$.

In [6], Ferret and Storme proved that increasing $h$ to $2 \sqrt{q}-1$ allows one Baer subgeometry in the minihyper.

Theorem 1.3 ([6, Theorem 5.9]) Let $F$ be a $\left\{\sum_{i=0}^{k-2} \epsilon_{i} \theta_{i}, \sum_{i=0}^{k-2} \epsilon_{i} \theta_{i-1} ; k-\right.$ $1, q\}$-minihyper, $q$ square, $q=p^{h}$, h even, $p$ prime, where $\sum_{i=0}^{k-2} \epsilon_{i} \leq \min \{2 \sqrt{q}-$ $\left.1, c_{p} q^{5 / 9}\right\}, c_{p}=2^{-1 / 3}, q \geq 2^{14}$, when $p=2,3$, and where $\sum_{i=0}^{k-2} \epsilon_{i} \leq \min \{2 \sqrt{q}-$ $\left.1, q^{6 / 9} /\left(1+q^{1 / 9}\right)\right\}, q \geq 2^{12}$, when $p>3$.

Then $F$ consists of the union of pairwise disjoint 
(1) $\epsilon_{k-2}$ spaces $\mathrm{PG}(k-2, q), \epsilon_{k-3}$ spaces $\mathrm{PG}(k-3, q), \ldots, \epsilon_{0}$ points, or

(2) one subgeometry $\mathrm{PG}(2 l+1, \sqrt{q})$, for some integer $l$ with $1 \leq l \leq \frac{k-2}{2}$, $\epsilon_{k-2}$ spaces $\mathrm{PG}(k-2, q), \ldots, \epsilon_{l+1}$ spaces $\mathrm{PG}(l+1, q), \epsilon_{l}-\sqrt{q}-1$ spaces $\mathrm{PG}(l, q), \epsilon_{l-1}$ spaces $\mathrm{PG}(l-1, q), \ldots, \epsilon_{0}$ points, or

(3) one subgeometry $\mathrm{PG}(2 l, \sqrt{q})$, for some integer $l$ with $1 \leq l \leq \frac{k-1}{2}$, $\epsilon_{k-2}$ spaces $\mathrm{PG}(k-2, q), \ldots, \epsilon_{l+1}$ spaces $\mathrm{PG}(l+1, q), \epsilon_{l}-1$ spaces $\mathrm{PG}(l, q), \epsilon_{l-1}-\sqrt{q}$ spaces $\mathrm{PG}(l-1, q), \epsilon_{l-2}$ spaces $\mathrm{PG}(l-2, q), \ldots, \epsilon_{0}$ points.

In this article, in Theorems 3.11 and 4.2, we give a characterisation of these minihypers with $h<\frac{q^{7 / 12}}{2}-\frac{q^{1 / 4}}{2}$ and $s=1$. In particular, we prove the following theorem.

Theorem 1.4 Let $F$ be a non-weighted $\left\{\epsilon_{1}(q+1)+\epsilon_{0}, \epsilon_{1} ; n, q\right\}$-minihyper, $q$ square, $q=p^{h}, p>3$ prime, $h \geq 2, q \geq 1217, n \geq 4$, where $\epsilon_{1}+\epsilon_{0}=$ $\eta\left(\sqrt{q}-q^{1 / 6}\right)<\frac{q^{7 / 12}}{2}-\frac{q^{1 / 4}}{2}$, then $F$ is the union of pairwise disjoint $A$ lines, $B$ isolated Baer subplanes $\mathrm{PG}(2, \sqrt{q})$ and $C$ Baer subgeometries $\mathrm{PG}(3, \sqrt{q})$, with $A+B+C(\sqrt{q}+1)=\epsilon_{1}$, and $\epsilon_{0}-B \sqrt{q}$ extra points.

\section{Preliminaries}

We explain formally the notion of removing a point $P$ from a weighted minihyper $(F, w)$. Suppose that $(F, w)$ is a weighted minihyper. With the notation $P \in(F, w)$, we always mean $w(P)>0$, equivalently $P \in F$.

Suppose that we have two weighted sets $\left(F_{1}, w_{1}\right)$ and $\left(F_{2}, w_{2}\right)$ in $\operatorname{PG}(n, q)$, where $w_{1}(P) \geq w_{2}(P)$ for all points $P$ of $\mathrm{PG}(n, q)$. Then we can define the new weighted set $(F, w)=\left(F_{1}, w_{1}\right)-\left(F_{2}, w_{2}\right)$ defined by the weight function $w$, with $w: \operatorname{PG}(n, q) \rightarrow \mathbb{N}: P \mapsto w(P)=w_{1}(P)-w_{2}(P)$. When the weights $w_{2}(P)$ of all the points $P$ of $\operatorname{PG}(n, q)$ are equal to zero or one, we simply write this difference as $(F, w)=\left(F_{1}, w_{1}\right)-F_{2}$.

For instance, suppose that $P \in(F, w)$ and define $w^{\prime}: \operatorname{PG}(n, q) \rightarrow \mathbb{N}$ : $w^{\prime}(R)=w(R)$ for any point $R \in \operatorname{PG}(n, q) \backslash\{P\}$ and $w^{\prime}(P)=w(P)-1$. Then $w^{\prime}$ determines a new set $F^{\prime}$ and $(F, w)-\{P\}$ is by definition $\left(F^{\prime}, w^{\prime}\right)$. This is the weighted minihyper in which the point $P$ is removed once from $(F, w)$. It is clear that $F^{\prime}=F \backslash\{P\}$ when $(F, w)$ is a non-weighted minihyper. 
We can easily extend the notion of removing points from $(F, w)$ to removing sets $M \subseteq F$ from $(F, w)$ by defining $w^{\prime}: \operatorname{PG}(n, q) \rightarrow \mathbb{N}: w^{\prime}(R)=w(R)$ for any point $R \in \mathrm{PG}(n, q) \backslash M$ and $w^{\prime}(P)=w(P)-1$ for $P \in M$.

Removing points or sets from a minihyper $(F, w)$ can, under certain circumstances, yield a minihyper as expected, as is shown in the next lemma.

Lemma 2.1 Let $(F, w)$ be a weighted $\left\{\epsilon_{1}(q+1)+\epsilon_{0}, \epsilon_{1} ; n, q\right\}$-minihyper, $n \geq$ 3 , with $2 \epsilon_{1}+\epsilon_{0}<q+2$, containing a line $L$. Then $(F, w)-L$ is a weighted $\left\{\left(\epsilon_{1}-1\right)(q+1)+\epsilon_{0}, \epsilon_{1}-1 ; n, q\right\}$-minihyper.

Proof. We have to show that $(F, w)-L$ is a weighted $\left\{\left(\epsilon_{1}-1\right)(q+1)+\right.$ $\left.\epsilon_{0}, \epsilon_{1}-1 ; n, q\right\}$-minihyper. The essential part is to show that any hyperplane intersects $(F, w)-L$ in at least $\epsilon_{1}-1$ points. So consider the line $L$ and an arbitrary hyperplane $\pi$. Since $|\pi \cap(F, w)| \geq \epsilon_{1}$, it follows immediately that $|\pi \cap((F, w)-L)| \geq \epsilon_{1}-1$ if $\pi$ intersects $L$ in exactly one point.

We are left with the case $L \subset \pi$. If $|\pi \cap(F, w)| \geq q+\epsilon_{1}$, then clearly, $|\pi \cap((F, w)-L)| \geq \epsilon_{1}-1$. So suppose that $|\pi \cap(F, w)|<q+\epsilon_{1}$.

Consider an $(n-3)$-dimensional space $\Omega$ in $\pi$ skew to $F$. The minihyper $(F, w)$ is projected from $\Omega$ on a weighted $\left\{\epsilon_{1}(q+1)+\epsilon_{0}, \epsilon_{1} ; 2, q\right\}$-minihyper $\left(F^{\prime}, w^{\prime}\right)$. The projection of $L$ is a line $L^{\prime}$ contained in $\left(F^{\prime}, w^{\prime}\right)$. By [8, Theorem 2.2], we can reduce the weight of every point of $L^{\prime}$ by one to obtain an $\left(\epsilon_{1}-1\right)$ fold blocking set $F^{\prime \prime}$ in this plane. But then $L^{\prime}$ is still blocked at least $\epsilon_{1}-1$ times. So $\pi$ is blocked at least $q+\epsilon_{1}$ times by $F$.

As we may consider minihypers containing no lines, the following lemma will provide information on their size. It is proved in [4] and it is a generalisation of a result from [1].

Lemma 2.2 A weighted $\{f, t ; 2, q\}$-minihyper $(B, w)$, with $1 \leq t<q-1$ and $q \geq 3$, contains a line or satisfies $f \geq t q+\sqrt{t q}+1$.

We first of all wish to characterise weighted $\left\{\epsilon_{1}(q+1)+\epsilon_{0}, \epsilon_{1} ; 3, q\right\}$ minihypers, $q$ square, having weighted points with total weight of the weighted points at most $\frac{2 \epsilon_{1}^{2}}{q}$ and where $\epsilon_{1}+\epsilon_{0}=\eta\left(\sqrt{q}-q^{1 / 6}\right)<\frac{q^{7 / 12}}{2}-\frac{q^{1 / 4}}{2}$, as a sum of $A$ lines, $B$ isolated Baer subplanes $\mathrm{PG}(2, \sqrt{q})$ and $C$ Baer subgeometries $\mathrm{PG}(3, \sqrt{q})$, where $A+B+C(\sqrt{q}+1)=\epsilon_{1}$, and $\epsilon_{0}-B \sqrt{q}$ extra points.

With an isolated Baer subplane $\mathrm{PG}(2 \sqrt{q})$ contained in $F$, we mean a Baer subplane $\operatorname{PG}(2, \sqrt{q})$ contained in $F$, but not contained in a 3-dimensional Baer subgeometry $\mathrm{PG}(3, \sqrt{q})$, completely contained in $F$. 
We will focus on the existence of the isolated Baer subgeometries $\mathrm{PG}(2, \sqrt{q})$ and the Baer subgeometries PG $(3, \sqrt{q})$ contained in $(F, w)$. To find Baer subgeometries completely contained in $(F, w)$, we will use a result of Barát and Storme [2]. This paper contains a lot of results on multiple (weighted) blocking sets in projective spaces, and we state a related result required in this article.

Theorem 2.3 Let $B$ be an s-fold blocking set in $\mathrm{PG}(2, q), q=p^{h}, p$ prime, $h \geq 1$, having points with multiplicities. Assume that $|B| \leq s(q+1)+c$ where

(1) $h>1, c<c_{p} q^{2 / 3}$ and $s<\min \left(c_{p} q^{1 / 6}, q^{1 / 4} / 2\right)$ where $c_{2}=c_{3}=2^{-1 / 3}$ and $c_{p}=1$ for $p>3$,

(2) $q=p^{2}, s<q^{1 / 4} / 2$ and $c<q^{3 / 4} / 2$,

and assume that $B$ has at least $(s-2)(q+\sqrt{q}+1)+16 \sqrt{q}+8 q^{1 / 4}$ simple points in (1) and at least $(s-2)(q+\sqrt{q}+1)+16 \sqrt{q}+16 q^{1 / 6}$ simple points in (2).

Then $B$ contains the sum of s Baer subplanes and/or lines.

This theorem is proved in exactly the same way as [2, Theorem 3.10], but using two new arguments. First of all, the $t(\bmod p)$ result on small weighted minimal $t$-fold blocking sets in $\mathrm{PG}(2, q), q=p^{h}, p$ prime, $h \geq 1[8$, Theorem 2.13], that is now known, has to be used. Secondly, by [8, Theorem 2.2 ], if a line $L$ is contained in the $s$-fold blocking set $B$, then we can reduce the weight of every point of $L$ by one to obtain an $(s-1)$-fold blocking set $F^{\prime \prime}$ in this plane. By using these two new arguments, the upper bound $s(q+1)+c-(s-1)(s-2) / 2$ in $[2$, Theorem 3.10] can be replaced by the upper bound $s(q+1)+c$.

Suppose that $(F, w)$ is a weighted minihyper in $\operatorname{PG}(n, q)$ and consider any subspace $\pi$ of $\operatorname{PG}(n, q)$. Then $(F, w) \cap \pi$ is the weighted minihyper $\left(F^{\prime}, w^{\prime}\right)$ induced in the subspace $\pi$, where $F^{\prime}:=F \cap \pi$ and $w^{\prime}$ is the function $w$ restricted to the points of $\pi$. The following theorem provides useful information on intersections of weighted minihypers with subspaces.

Theorem 2.4 ([4, Result 2.9]) Let $(F, w)$ be a weighted $\left\{\sum_{i=0}^{n-1} \epsilon_{i} \theta_{i}, \sum_{i=0}^{n-1} \epsilon_{i} \theta_{i-1}\right.$; $n, q\}$-minihyper satisfying $n \geq 1, \sum_{i=0}^{n-1} \epsilon_{i}=h \leq q$. Then every $r$-space $\pi_{r}, 1 \leq r \leq n$, not contained in $(F, w)$, intersects $(F, w)$ in a weighted $\left\{\sum_{i=0}^{r-1} m_{i} \theta_{i}, \sum_{i=0}^{r-1} m_{i} \theta_{i-1} ; r, q\right\}$-minihyper $(F, w) \cap \pi_{r}$, satisfying $\sum_{i=0}^{r-1} m_{i} \leq$ $h$. 
Lemma 2.5 Let $(F, w)$ be a weighted $\left\{\epsilon_{1}(q+1)+\epsilon_{0}, \epsilon_{1} ; n, q\right\}$-minihyper, with $\epsilon_{1}+\epsilon_{0}<\frac{q^{7 / 12}}{2}-\frac{q^{1 / 4}}{2}$, containing no lines and having at most $q^{1 / 6} / 2$ multiple points. If a plane $\pi$ intersects $(F, w)$ in a weighted $\left\{m_{1}(q+1)+m_{0}, m_{1} ; 2, q\right\}$ minihyper, with $m_{1} \geq 1$, then $(F, w) \cap \pi$ contains a sum of $m_{1}$ Baer subplanes.

Proof. By Theorem 2.4, we know that $m_{1}+m_{0} \leq \epsilon_{1}+\epsilon_{0}<\frac{q^{7 / 12}}{2}-\frac{q^{1 / 4}}{2}$. The intersection of $\pi$ with $F$ does not contain lines, since $F$ does not contain lines, so $|\pi \cap(F, w)| \geq m_{1} q+\sqrt{m_{1} q}+1$ by Lemma 2.2 , which implies $\sqrt{m_{1} q}+1<$ $\frac{q^{7 / 12}}{2}-\frac{q^{1 / 4}}{2}$. Hence, $m_{1}<q^{1 / 6} / 2$. By Theorem 2.3, $\pi \cap(F, w)$ contains a sum of $m_{1}$ Baer subplanes.

\section{Minihypers in three dimensions}

To obtain the desired characterisation, we will use an inductive argument on the dimension $n \geq 3$. In this inductive step, we will require a characterisation of weighted minihypers in three dimensions, which we will obtain in this section.

The following theorem, which is an improvement of [2, Theorem 3.1], also plays a crucial role.

Theorem 3.1 ([9, Theorem 3.1]) Let $B$ be a t-fold blocking set in $\mathrm{PG}(n, q)$, $q=p^{h}, p$ prime, $q \geq 661, n \geq 3$, of size $|B|<t q+c_{p} q^{2 / 3}$, with $c_{2}=c_{3}=$ $2^{-1 / 3}, c_{p}=1$ when $p>3$, and with $t<c_{p} q^{1 / 6} / 2$. Then $B$ contains a union of $t$ pairwise disjoint lines and/or Baer subplanes.

We assume that $(F, w)$ is a weighted $\left\{\epsilon_{1}(q+1)+\epsilon_{0}, \epsilon_{1} ; 3, q\right\}$-minihyper, $q$ square, $q=p^{h}, p>3$ prime, $h \geq 2, q \geq 1217$, where the total weight of the multiple points is at most $\frac{2 \epsilon_{1}^{2}}{q}$ and with $\epsilon_{1}+\epsilon_{0}=\eta\left(\sqrt{q}-q^{1 / 6}\right)<\frac{q^{7 / 12}}{2}-\frac{q^{1 / 4}}{2}$, so $\eta<\frac{q^{1 / 12}}{2}$, and we assume that $(F, w)$ does not contain a line of $\operatorname{PG}(3, q)$. The preceding Theorem 3.1 characterises these minihypers for $\epsilon_{1}<q^{1 / 6} / 2$, so from now on, we assume that $\epsilon_{1} \geq q^{1 / 6} / 2$.

Remark 3.2 As indicated in the preceding paragraph, we assume that $q=$ $p^{h}, q$ square, $p>3$ prime, $h \geq 2$, and that $q \geq 1217$. The condition $q \geq 1217$ follows from the inequality at the end of the proof of Lemma 3.9.

The other inequalities in the proofs are valid for $q \geq 1217$.

In the main theorems, we repeat that $q=p^{h}, p>3$ prime, $h \geq 2$, and that $q \geq 1217$, to give the correct statements of the theorems. 
Projecting the minihyper $(F, w)$ from a point $R \notin F$ onto a plane gives a weighted $\epsilon_{1}$-fold blocking set $B$ in this plane. This set $B$ can contain lines, and we will distinguish two cases.

First we assume that $B$ does not contain a line.

Lemma 3.3 If $B$ does not contain a line, then $\epsilon_{1}<\frac{q^{1 / 6}}{2}$ and $(F, w)$ is an $\epsilon_{1}$-fold blocking multiset containing a sum of $\epsilon_{1}$ Baer subplanes and lines.

Proof. The set $B$ is a weighted $\epsilon_{1}$-fold blocking set in $\operatorname{PG}(2, q)$ of size $\epsilon_{1}(q+1)+\epsilon_{0}<\epsilon_{1} q+\frac{q^{7 / 12}}{2}-\frac{q^{1 / 4}}{2}$, containing no lines. Lemma 2.2 implies that $\epsilon_{1}<\frac{q^{1 / 6}}{2}$. In this case, there are no multiple points since $\frac{2 \epsilon_{1}^{2}}{q}<1$.

So $(F, w)$ is an $\epsilon_{1}$-fold blocking set satisfying the conditions of Theorem 3.1, and by assumption not containing lines. Using Theorem 3.1, we conclude that $(F, w)$ contains a union of $\epsilon_{1}$ pairwise disjoint Baer subplanes. Hence, $(F, w)$ is the sum of $\epsilon_{1}$ Baer subplanes and points.

An upper bound on the number of secants to $F$ through a point $R$, not in $F$, will be very useful. The next lemma yields such an upper bound.

Lemma 3.4 There exists in $\mathrm{PG}(3, q)$ a point not in $F$ lying on at most $\frac{\epsilon_{1}^{2}+2 \eta^{2}}{2}$ secants to $F$, containing at least two points of $F$ of weight one.

Proof. We count the number of points of $\mathrm{PG}(3, q)$, not contained in $F$, on secants to $F$ through two points of weight one. Here, $|F| \leq \epsilon_{1} q+\eta \sqrt{q}$, but we subtract $\frac{q^{1 / 6}}{2}$ from $|F|$, since there can be up to $\frac{2 \epsilon_{1}^{2}}{q}<\frac{q^{1 / 6}}{2}$ multiple points in $(F, w)$ :

$$
\begin{aligned}
& \left(\epsilon_{1} q+\eta \sqrt{q}-\frac{q^{1 / 6}}{2}\right)\left(\epsilon_{1} q+\eta \sqrt{q}-\frac{q^{1 / 6}}{2}-1\right) \frac{(q-1)}{2} \\
\leq & \frac{\epsilon_{1}^{2} q^{3}+2 \eta \epsilon_{1} q^{2} \sqrt{q}+\eta^{2} q^{2}-\epsilon_{1} q^{2}-\eta q \sqrt{q}-\epsilon_{1}^{2} q^{2}-2 \eta \epsilon_{1} q \sqrt{q}-\eta^{2} q+\epsilon_{1} q+\eta \sqrt{q}}{2} \\
\leq & \frac{\epsilon_{1}^{2} q^{3}+2 \eta \epsilon_{1} q^{2} \sqrt{q}+\eta^{2} q^{2}-\epsilon_{1}^{2} q^{2}-2 \eta \epsilon_{1} q \sqrt{q}-\eta^{2} q}{2} .
\end{aligned}
$$

Since we can assume that $\epsilon_{1} \geq q^{1 / 6} / 2$, also $\epsilon_{1} \geq \eta$, so the upper bound further simplifies to

$$
\frac{\epsilon_{1}^{2} q^{3}+2 \epsilon_{1} \eta q^{2} \sqrt{q}}{2} \leq \frac{\epsilon_{1}^{2} q^{3}+2 \eta^{2} q^{3}}{2},
$$


where the last inequality follows from $\epsilon_{1} \leq \eta \sqrt{q}$.

There are $\theta_{3}-|F| \geq q^{3}$ points in $\operatorname{PG}(3, q)$ not contained in $F$, hence, we find a point $R$, not in $F$, lying on at most $\frac{\epsilon_{1}^{2}+2 \eta^{2}}{2}$ such secants to $F$.

Lemma 3.5 If $B$ contains at least one line, then $\sqrt{q}-q^{1 / 6} \leq \epsilon_{1}$.

Proof. Consider a point $R$ of $\mathrm{PG}(3, q) \backslash F$ lying on at most $\frac{\epsilon_{1}^{2}+2 \eta^{2}}{2}$ secants to $F$, containing at least two simple points of $F$. The minihyper $F$ is projected from $R$ onto a weighted point set in a plane containing a line $L$. The plane $\langle R, L\rangle$ intersects $F$ in at least a 1-fold blocking set since $|\langle R, L\rangle \cap F| \geq$ $q+1$ (Theorem 2.4). So Lemma 2.5 implies that $\langle R, L\rangle \cap F$ contains a Baer subplane having a Baer subline on a line through $R$. This Baer subline has at most $\frac{q^{1 / 6}}{2}$ distinct multiple points of $F$, so is counted at least $\frac{1}{2}\left(\sqrt{q}-\frac{q^{1 / 6}}{2}\right)^{2}$ times as a secant in the previous lemma. This number must be smaller than or equal to the total number of such secants to $F$ through $R$, so

$$
\begin{gathered}
\left(\sqrt{q}-\frac{q^{1 / 6}}{2}\right)^{2} \leq \epsilon_{1}^{2}+2 \eta^{2} \\
\Leftrightarrow q-\sqrt{q} q^{1 / 6}+\frac{q^{1 / 3}}{4}-q^{1 / 6} \leq \epsilon_{1}^{2}, \quad \text { since } \eta<\frac{q^{1 / 12}}{2} \\
\Rightarrow\left(\sqrt{q}-q^{1 / 6}\right)^{2} \leq q-\sqrt{q} q^{1 / 6}+\frac{q^{1 / 3}}{4}-q^{1 / 6} \leq \epsilon_{1}^{2} .
\end{gathered}
$$

This last equation holds if $q \geq 4$ and then we have the assertion.

From now on, we assume that $\epsilon_{1} \geq \sqrt{q}-q^{1 / 6}$.

Since $\left(\epsilon_{1}^{2}+2 \eta^{2}\right) / 2 \leq \epsilon_{1}^{2}$ and since we will need in Lemma 3.8 a lot of points $R$, not in $F$, lying on a small number of secants to $F$, we will look for points $R$, not in $F$, lying on at most $\epsilon_{1}^{2}$ secants to $F$ containing at least two simple points of $F$.

Lemma 3.6 Let $R$ be a point of $\mathrm{PG}(3, q) \backslash F$ lying on at most $\epsilon_{1}^{2}$ secants to $F$, containing at least two simple points of $F$. Then $R$ lies on a line containing a Baer subline of $F$ which is contained in at least $\frac{\epsilon_{1}}{2 \eta^{2}}-\frac{q^{1 / 6}}{4 \eta^{2}}$ Baer subplanes of $F$, containing at least $\frac{\epsilon_{1} q}{2 \eta^{2}}-\frac{q^{7 / 6}}{4 \eta^{2}}+\sqrt{q}+1$ points of $F$.

Proof. The projection of $F$ from $R$ is a weighted $\epsilon_{1}$-fold blocking set $B$ in a plane, containing lines. Let $x$ be the number of lines contained in $B$, 
where some lines can be counted more than once in this weighted $\epsilon_{1}$-fold blocking set. It follows from Lemma 2.1 that the $x$ lines contained in $B$ can be removed from $B$ to obtain a new weighted $\left(\epsilon_{1}-x\right)$-fold blocking set $B^{\prime}$, containing no lines. Denote $\epsilon_{1}-x$ by $\epsilon_{1}^{\prime}$. By Lemma 3.3, for an $\epsilon_{1}^{\prime}$-fold blocking set $B$ of size $\epsilon_{1}^{\prime}(q+1)+\epsilon_{0}$ without lines, necessarily $\epsilon_{1}^{\prime}<\frac{q^{1 / 6}}{2}$, so $B$ must contain at least $\epsilon_{1}-\epsilon_{1}^{\prime}>\epsilon_{1}-\frac{q^{1 / 6}}{2}$ lines.

For each such line $L \subset B$, let $m_{1}$ be its multiplicity as a line in the weighted set $B$. Then the plane $\langle R, L\rangle$ intersects $F$ in an $\left\{m_{1}(q+1)+\right.$ $\left.m_{0}, m_{1} ; 2, q\right\}$-minihyper, with $m_{1}+m_{0} \leq \epsilon_{1}+\epsilon_{0}<\frac{q^{7 / 12}}{2}-\frac{q^{1 / 4}}{2}$ (Theorem 2.4). This plane $\langle R, L\rangle$ contains $m_{1}$ Baer subplanes of $F$ (Lemma 2.5), and, for each such Baer subplane in $\langle R, L\rangle \cap F$, there is a line through $R$ containing a Baer subline of this Baer subplane.

A Baer subline is counted at least $\frac{1}{2}\left(\sqrt{q}-\frac{q^{1 / 6}}{2}\right)^{2}$ times as a secant in Lemma 3.4. The point $R$ lies on at most $\epsilon_{1}^{2} \leq \eta^{2}\left(q-q^{2 / 3}+\frac{q^{1 / 3}}{4}\right)$ secants, hence $R$ lies on at most $2 \eta^{2}$ different lines containing a Baer subline of $F$. There are at least $\epsilon_{1}-\frac{q^{1 / 6}}{2}$ Baer sublines, in Baer subplanes of $F$, on lines through $R$. So some Baer subline lies in at least $\frac{\epsilon_{1}}{2 \eta^{2}}-\frac{q^{1 / 6}}{4 \eta^{2}}$ Baer subplanes of $F$. These Baer subplanes of $F$ contain at least $\frac{\epsilon_{1} q}{2 \eta^{2}}-\frac{q^{7 / 6}}{4 \eta^{2}}+\sqrt{q}+1$ points of $F$.

Remark 3.7 We will denote these Baer subplanes, contained in F, through a common Baer subline on a line through $R$ as flags of Baer subplanes corresponding to $R$. The next lemma shows that we can find several flags which leads to the fact that they must intersect each other in a certain minimum number of points.

Lemma 3.8 (1) There are more than $8 \eta^{2}$ points $R$ of $\mathrm{PG}(3, q) \backslash F$, defining different flags of Baer subplanes.

(2) There are two such flags intersecting each other in at least $\frac{q}{16 \eta^{2}}\left(\frac{\epsilon_{1}}{2 \eta^{2}}-\right.$ $\left.\frac{q^{1 / 6}}{4 \eta^{2}}\right)$ points of $F$.

Proof. (1) We find the required points one by one. There exists at least one such point (Lemmas 3.4 and 3.6).

Suppose that we have already found $8 \eta^{2}$ points $R$ not in $F$ with a corresponding flag of Baer subplanes, as in the previous lemma. Is there another point of $\mathrm{PG}(3, q) \backslash F$ lying on at most $\epsilon_{1}^{2}$ secants to $F$, containing at least 
two simple points of $F$ ? The number of points in these $8 \eta^{2}$ flags, in the corresponding planes $\operatorname{PG}(2, q)$, is at most

$$
8 \eta^{2}\left(\left(\frac{\epsilon_{1}}{2 \eta^{2}}-\frac{q^{1 / 6}}{4 \eta^{2}}\right) q^{2}+q+1\right)=4 \epsilon_{1} q^{2}-2 q^{1 / 6} q^{2}+8 \eta^{2}(q+1) .
$$

We count these points in the corresponding planes $\mathrm{PG}(2, q)$ to assure that the new flag is different from the ones we already have. There are at least $q^{3}+q^{2}+q+1-\epsilon_{1}(q+1)-\epsilon_{0}-4 \epsilon_{1} q^{2}+2 q^{1 / 6} q^{2}-8 \eta^{2}(q+1)$ points in $\mathrm{PG}(3, q)$ not in $F$ and not in the extended flags. If all these points lie on more than $\epsilon_{1}^{2}$ secants to $F$, then the number of incidences on the remaining secants is larger than $\left(\epsilon_{1}^{2} q^{3}+2 \eta^{2} q^{3}\right) / 2$, the total number of incidences on secants to $F$ we had in Lemma 3.3. So there is still another point $P \notin F$ on at most $\epsilon_{1}^{2}$ secants to $F$.

(2) Take $8 \eta^{2}$ such points $R$ and suppose that the union of the $\frac{\epsilon_{1}}{2 \eta^{2}}-\frac{q^{1 / 6}}{4 \eta^{2}}$ Baer subplanes through the Baer subline of a flag corresponding to a point $R$ share for two such points $R$ and $R^{\prime}$ at most $\frac{q}{16 \eta^{2}}\left(\frac{\epsilon_{1}}{2 \eta^{2}}-\frac{q^{1 / 6}}{4 \eta^{2}}\right)$ points of $F$. Then

$$
\begin{aligned}
|F| & \geq \sum_{i=1}^{8 \eta^{2}}\left(\frac{\epsilon_{1} q}{2 \eta^{2}}-\frac{q^{7 / 6}}{4 \eta^{2}}+\sqrt{q}+1-(i-1) \frac{q}{16 \eta^{2}}\left(\frac{\epsilon_{1}}{2 \eta^{2}}-\frac{q^{1 / 6}}{4 \eta^{2}}\right)\right) \\
& \geq 8 \eta^{2}\left(\frac{\epsilon_{1} q}{2 \eta^{2}}-\frac{q^{7 / 6}}{4 \eta^{2}}+\sqrt{q}+1\right)+\frac{\left(8 \eta^{2}\right)^{2}}{2} \frac{q}{16 \eta^{2}}\left(\frac{\epsilon_{1}}{2 \eta^{2}}-\frac{q^{1 / 6}}{4 \eta^{2}}\right) \\
& \geq 3 \epsilon_{1} q-\frac{3}{2} q^{7 / 6}+8 \eta^{2}(\sqrt{q}+1) .
\end{aligned}
$$

This is false since $\epsilon_{1} \geq \sqrt{q}-q^{1 / 6}$.

We have found different points $R$ and $R^{\prime}$ with a corresponding flag of Baer subplanes. We now build with them a Baer subgeometry PG $(3, \sqrt{q})$ contained in $F$.

Lemma 3.9 The minihyper $F$ contains a Baer subgeometry $\mathrm{PG}(3, \sqrt{q})$ if $\epsilon_{1} \geq \sqrt{q}-q^{1 / 6}$.

Proof. Let $R$ and $R^{\prime}$ be two points of $\mathrm{PG}(3, q) \backslash F$ corresponding with a flag of $\frac{\epsilon_{1}}{2 \eta^{2}}-\frac{q^{1 / 6}}{4 \eta^{2}}$ Baer subplanes of $F$, where these two flags share at least $\frac{q}{16 \eta^{2}}\left(\frac{\epsilon_{1}}{2 \eta^{2}}-\frac{q^{1 / 6}}{4 \eta^{2}}\right)$ points. Denote those two flags by $f_{R}$ and $f_{R^{\prime}}$. Then some 
Baer subplane $\pi_{R^{\prime}}$ of $f_{R^{\prime}}$ shares at least $\frac{q}{16 \eta^{2}}$ points with the Baer subplanes of $f_{R}$. If this Baer subplane $\pi_{R^{\prime}}$ shares at most two points with every Baer subplane of $f_{R}$, then $\frac{q}{16 \eta^{2}} \leq 2\left(\frac{\epsilon_{1}}{2 \eta^{2}}-\frac{q^{1 / 6}}{4 \eta^{2}}\right)$, which is false since $\epsilon_{1}<\frac{q^{7 / 12}}{2}-\frac{q^{1 / 4}}{2}$. So this Baer subplane $\pi_{R^{\prime}}$ shares a Baer subline with some Baer subplane of $f_{R}$. Denote by $l$ the Baer subline of the flag $f_{R}$. This Baer subplane $\pi_{R^{\prime}}$ cannot pass through $l$, since then this Baer subplane $\pi_{R^{\prime}}$ only shares this subline $l$ with all these Baer subplanes of the flag $f_{R}$, but $\frac{q}{16 \eta^{2}}>\sqrt{q}+1$.

We wish to find a lower bound on the number of Baer subplanes of $f_{R}$, sharing a Baer subline with the Baer subplane $\pi_{R^{\prime}}$. Two distinct Baer sublines share at most two points. We subtract two for every of the $\frac{\epsilon_{1}}{2 \eta^{2}}-\frac{q^{1 / 6}}{4 \eta^{2}}$ Baer subplanes of $f_{R}$ from $\frac{q}{16 \eta^{2}}$ and then divide by $\sqrt{q}-1$. The quotient is at least $\frac{q^{1 / 2}}{16 \eta^{2}}+\frac{1}{16 \eta^{2}}-\frac{1}{\eta}$, hence this Baer subplane $\pi_{R^{\prime}}$ shares a Baer subline with at least $\frac{q^{1 / 2}}{16 \eta^{2}}+\frac{1}{16 \eta^{2}}-\frac{1}{\eta}$ Baer subplanes of $f_{R}$. Take this Baer subplane $\pi_{R^{\prime}}$ and consider a Baer subplane $\pi_{R}$ of the flag $f_{R}$ which shares a Baer subline with $\pi_{R^{\prime}}$. Together they define a Baer subgeometry $\Omega$ isomorphic to $\mathrm{PG}(3, \sqrt{q})$. Every Baer subplane of $f_{R}$ intersecting $\pi_{R^{\prime}}$ in a Baer subline shares $l$ and this Baer subline with $\Omega$. Two intersecting Baer sublines define a Baer subplane in a unique way, so these Baer subplanes then lie completely in this Baer subgeometry $\Omega$.

Consider an arbitrary Baer subplane $\pi$ of $\Omega$ not through $l$. Then $\pi$ shares at least $\frac{q^{1 / 2}}{16 \eta^{2}}+\frac{1}{16 \eta^{2}}-\frac{1}{\eta}$ Baer sublines with $F$, so shares at least $\frac{q}{16 \eta^{2}}+\frac{q^{1 / 2}}{16 \eta^{2}}-$ $\frac{q^{1 / 2}}{\eta}+1$ points with $F$. Consider the plane over $\mathbb{F}_{q}$ of this Baer subplane $\pi$. This plane intersects $F$ in an $\left\{m_{1}(q+1)+m_{0}, m_{1} ; 2, q\right\}$-minihyper, with $m_{1}+m_{0} \leq \epsilon_{1}+\epsilon_{0}=\eta\left(q^{1 / 2}-q^{1 / 6}\right)$, which contains $m_{1}$ Baer subplanes (Lemma 2.5). Suppose this Baer subplane $\pi$ is not contained in $F$. It contains already at least $\frac{q}{16 \eta^{2}}+\frac{q^{1 / 2}}{16 \eta^{2}}-\frac{q^{1 / 2}}{\eta}+1$ points of $F$. By [3, Lemma 4.4], we have that

$$
|\pi \cap F| \leq m_{0}+m_{1}(\sqrt{q}+1) \leq 2 \eta\left(q^{1 / 2}-q^{1 / 6}\right),
$$

where the upper bound is obtained in the following way. First of all, $m_{0}+$ $m_{1} \leq \eta\left(q^{1 / 2}-q^{1 / 6}\right)$. Secondly, every one of the $m_{1}$ Baer subplanes contained in the $\left\{m_{1}(q+1)+m_{0}, m_{1} ; 2, q\right\}$-minihyper has size $q+\sqrt{q}+1$ and contributes $\sqrt{q}+1$ to the sum $m_{1}+m_{0}$. Hence, also $m_{1} \sqrt{q} \leq \eta\left(q^{1 / 2}-q^{1 / 6}\right)$.

But $\frac{q}{16 \eta^{2}}+\frac{q^{1 / 2}}{16 \eta^{2}}-\frac{q^{1 / 2}}{\eta}+1>2 \eta\left(q^{1 / 2}-q^{1 / 6}\right)$ if $q \geq 1217$, so this Baer subplane $\pi$ lies completely in $F$. As a consequence, this Baer subgeometry $\Omega$ defined by $\pi_{R}$ and $\pi_{R^{\prime}}$ lies completely in $F$. 
Lemma 3.10 Let $F$ be an $\left\{\epsilon_{1}(q+1)+\epsilon_{0}, \epsilon_{1} ; 3, q\right\}$-minihyper, with $2 \epsilon_{1}+$ $\epsilon_{0}<q+2$, containing a subgeometry $\mathrm{PG}(3, \sqrt{q})$. Then $F \backslash \mathrm{PG}(3, \sqrt{q})$ is an $\left\{\left(\epsilon_{1}-\sqrt{q}-1\right)(q+1)+\epsilon_{0}, \epsilon_{1}-\sqrt{q}-1 ; 3, q\right\}$-minihyper.

Proof. A plane $\pi$ either intersects a Baer subgeometry $\operatorname{PG}(3, \sqrt{q})$ in a subline $\mathrm{PG}(1, \sqrt{q})$ or a subplane $\mathrm{PG}(2, \sqrt{q})$. We only have to discuss the case that $\pi \cap \mathrm{PG}(3, \sqrt{q})$ is a subplane $\mathrm{PG}(2, \sqrt{q})$ of size $q+\sqrt{q}+1$.

If $\pi$ still contains $\epsilon_{1}-\sqrt{q}-1$ other points of $F$, then removing this Baer subgeometry $\mathrm{PG}(3, \sqrt{q})$ from $F$ causes no problem for the plane $\pi$. So from now on, we assume that $q+\sqrt{q}+1 \leq|\pi \cap F|<q+\epsilon_{1}$.

We select a point $R$ of $\pi \backslash F$. Project $\pi$ and $F$ from $R$ onto a plane. Then we obtain an $\epsilon_{1}$-fold blocking multiset $B$ in this plane containing a line $L$, which is the projection of $\pi \cap F$. By [8, Theorem 2.2], we can reduce the weight of every point of $L$ by one to obtain an $\left(\epsilon_{1}-1\right)$-fold blocking set $B^{\prime}$ in this plane. But then $L$ is still blocked at least $\epsilon_{1}-1$ times by $B^{\prime}$. So $\pi$ is blocked at least $q+\epsilon_{1}$ times by $F$.

Theorem 3.11 Let $F$ be a weighted $\left\{\epsilon_{1}(q+1)+\epsilon_{0}, \epsilon_{1} ; 3, q\right\}$-minihyper, $q=p^{h}$, $q$ square, $p>3$ prime, $h \geq 2$, having weighted points with total weight at most $\frac{2 \epsilon_{1}^{2}}{q}$ and where $\epsilon_{1}+\epsilon_{0}=\eta\left(\sqrt{q}-q^{1 / 6}\right)<\frac{q^{7 / 12}}{2}-\frac{q^{1 / 4}}{2}$, then $F$ contains a sum of $A$ lines, $B$ isolated Baer subplanes $\mathrm{PG}(2, \sqrt{q})$ and $C$ Baer subgeometries $\mathrm{PG}(3, \sqrt{q})$, where $A+B+C(\sqrt{q}+1)=\epsilon_{1}$, and $\epsilon_{0}-B \sqrt{q}$ extra points.

Proof. If $\epsilon_{1}<q^{1 / 6} / 2$, this is the result mentioned in Theorem 3.1, so from now on assume that $\epsilon_{1} \geq q^{1 / 6} / 2$.

If $F$ contains $A$ lines, we can remove these lines from $F$, and then apply the arguments to $F$ minus these $A$ lines (Lemma 2.1). We denote the minihyper that remains again by $F$. Let $R$ be a point not in the minihyper $F$ on at most $\frac{\epsilon_{1}^{2}+2 \eta^{2}}{2}$ secants to $F$, containing at least two points of $F$ of weight one (Lemma 3.4). Projecting $F$ from $R$ onto a plane not through $R$ gives a weighted $\epsilon_{1}$-fold blocking set $B$ in this plane. If $B$ does not contain lines, Lemma 3.3 states that $F$ is the sum of $\epsilon_{1}$ lines and Baer subplanes $\mathrm{PG}(2, \sqrt{q})$, and possibly some extra points. If $B$ does contain lines, we find a Baer subgeometry $\mathrm{PG}(3, \sqrt{q})$ contained in $F$, which can be removed from $F$ to obtain a new $\left\{\left(\epsilon_{1}-\sqrt{q}-1\right)(q+1)+\epsilon_{0}, \epsilon_{1}-\sqrt{q}-1 ; 3, q\right\}$-minihyper, see Lemma 3.10. Repeating the previous arguments with this minihyper gives us the assertion. 


\section{Larger dimensions}

We now characterise non-weighted $\left\{\epsilon_{1}(q+1)+\epsilon_{0}, \epsilon_{1} ; n, q\right\}$-minihypers $F, q$ square, $q=p^{h}, p>3$ prime, $h \geq 2, q \geq 1217, n \geq 4$, where $\epsilon_{1}+\epsilon_{0}=$ $\eta\left(\sqrt{q}-q^{1 / 6}\right)<\frac{q^{7 / 12}}{2}-\frac{q^{1 / 4}}{2}$, by induction on the dimension $n$. We suppose that every $\left\{\epsilon_{1}(q+1)+\epsilon_{0}, \epsilon_{1} ; n-1, q\right\}$-minihyper, with $n \geq 4$, is a pairwise disjoint union of $A$ lines, $B$ isolated Baer subplanes $\mathrm{PG}(2, \sqrt{q})$ and $C$ Baer subgeometries $\mathrm{PG}(3, \sqrt{q})$, with $A+B+C(\sqrt{q}+1)=\epsilon_{1}$, and $\epsilon_{0}-B \sqrt{q}$ extra points. As in the 3 -dimensional case, we start by using Lemma 2.1 to remove the lines contained in $F$.

We want to project $F$ onto a hyperplane in such a way that the number of multiple points appearing in the projection is as small as possible.

Lemma 4.1 For $n=4$, there is a point $R \notin F$ lying on at most $\frac{\epsilon_{1}^{2}}{q}$ secants to $F$. In larger dimensions $n$, there are points $R \notin F$ only lying on tangents to $F$.

Proof. The number of points on secants to $F$ is at most

$$
\frac{\left(\epsilon_{1}(q+1)+\epsilon_{0}\right)^{2}}{2}(q-1)=\frac{\epsilon_{1}\left(q^{3}+q^{2}-q-1\right)+2 \epsilon_{1} \epsilon_{0}\left(q^{2}-1\right)+\epsilon_{0}^{2}(q-1)}{2} .
$$

Now $\epsilon_{1} \epsilon_{0}, \epsilon_{0}^{2}<\frac{q^{7 / 6}}{4}$. For $n \geq 5$, this number is smaller than the number of points in $\mathrm{PG}(n, q) \backslash F$. In this case, there exists at least one point $R$ only lying on tangents to $F$.

For $n=4$, we divide by $q^{4}+q^{3} \leq \theta_{4}-|F|$. This gives a point $R$ lying on at most

$$
\frac{\epsilon_{1}^{2}}{2 q}+\frac{2 q^{7 / 6}\left(q^{2}-1\right) / 2+q^{7 / 6}(q-1) / 2}{2\left(q^{4}+q^{3}\right)} \leq \frac{\epsilon_{1}^{2}}{2 q}+\frac{1}{2 q^{5 / 6}}
$$

secants to $F$. Either $\frac{\epsilon_{1}^{2}}{2 q}+\frac{1}{2 q^{5 / 6}}<1$ and then $R$ lies on zero secants to $F$ or either $\frac{\epsilon_{1}^{2}}{2 q}+\frac{1}{2 q^{5 / 6}} \geq 1$, then $\frac{\epsilon_{1}^{2}}{2 q} \geq \frac{1}{2 q^{5 / 6}}$. In both cases, $\frac{\epsilon_{1}^{2}}{q}$ can be used as an upper bound on the number of secants to $F$ through $R$.

In the case of $n=4$, projecting from a point $R \notin F$ as in the previous lemma gives a weighted $\left\{\epsilon_{1}(q+1)+\epsilon_{0}, \epsilon_{1} ; n-1, q\right\}$-minihyper with the total weight of the multiple points at most $\frac{2 \epsilon_{1}^{2}}{q}$. This explains the bound in Theorem 3.11. 
Theorem 4.2 Let $F$ be a non-weighted $\left\{\epsilon_{1}(q+1)+\epsilon_{0}, \epsilon_{1} ; n, q\right\}$-minihyper, $q$ square, $q=p^{h}, p>3$ prime, $h \geq 2, q \geq 1217, n \geq 4$, where $\epsilon_{1}+\epsilon_{0}=$ $\eta\left(\sqrt{q}-q^{1 / 6}\right)<\frac{q^{7 / 12}}{2}-\frac{q^{1 / 4}}{2}$, then $F$ is the union of pairwise disjoint $A$ lines, $B$ isolated Baer subplanes $\mathrm{PG}(2, \sqrt{q})$ and $C$ Baer subgeometries $\mathrm{PG}(3, \sqrt{q})$, with $A+B+C(\sqrt{q}+1)=\epsilon_{1}$, and $\epsilon_{0}-B \sqrt{q}$ extra points.

Proof. We prove this result by induction on $n$. Theorem 3.11 proves this theorem for $n=3$. If $F$ contains lines, these lines can be removed from $F$ (Lemma 2.1); so we assume that $F$ does not contain any lines.

Project $F$ from a point $R$, lying only on tangents to $F$ or on at most $\epsilon_{1}^{2} / q$ secants to $F$ if $n=4$, onto a hyperplane $\pi$ not through $R$. We obtain a (weighted if $n=4)\left\{\epsilon_{1}(q+1)+\epsilon_{0}, \epsilon_{1} ; n-1, q\right\}$-minihyper $F^{\prime}$ which is the sum of $A^{\prime}$ lines, $B^{\prime}$ isolated Baer subplanes $\mathrm{PG}(2, \sqrt{q})$ and $C^{\prime}$ Baer subgeometries $\mathrm{PG}(3, \sqrt{q})$, with $A^{\prime}+B^{\prime}+C^{\prime}(\sqrt{q}+1)=\epsilon_{1}$, and $\epsilon_{0}-B \sqrt{q}$ points.

Case I: assume that $F^{\prime}$ contains a line $L$.

The plane $\langle R, L\rangle$ intersects $F$ in at least a 1-fold blocking set, which contains a Baer subplane, since by assumption, $F$ does not contain lines (Lemma 2.5).

Since $\langle R, L\rangle$ shares a Baer subplane with $F, R$ lies on a Baer subline to this Baer subplane, but then $R$ lies on a $(\sqrt{q}+1)$-secant to $F$, which is false for $n>4$. For $n=4$, this line is projected onto a point of $F^{\prime}$ with weight up to $\sqrt{q}+1>\frac{q^{1 / 6}}{2}>\frac{2 \epsilon_{1}^{2}}{q}$, which is false. So this case cannot occur.

Case II: assume that $F^{\prime}$ contains an isolated Baer subplane $\mathrm{PG}(2, \sqrt{q})$.

Denote this Baer subplane PG $(2, \sqrt{q})$ by $\omega$. The 3 -space $\langle R, \omega\rangle$ intersects $F$ in an $\left\{m_{1}(q+1)+m_{0}, m_{1} ; 3, q\right\}$-minihyper, with $m_{1} \geq 1$ (Lemma 2.4), so $\langle R, \omega\rangle \cap F$ contains by the induction hypothesis on $n$ the union of points, isolated Baer subgeometries $\mathrm{PG}(2, \sqrt{q})$ and Baer subgeometries $\mathrm{PG}(3, \sqrt{q})$, which are all pairwise disjoint. Assume $\langle R, \omega\rangle$ contains a Baer subgeometry $\mathrm{PG}(3, \sqrt{q})$ and consider the conjugate point $R^{\sqrt{q}}$ of $R$ w.r.t. $\mathrm{PG}(3, \sqrt{q})$. The line $R R^{\sqrt{q}}$ intersects $\mathrm{PG}(3, \sqrt{q})$ in a Baer subline, which is false since the projection of this Baer subline would lead to a projected point in $F^{\prime}$ with weight at least $\sqrt{q}+1$. So $\langle R, \omega\rangle \cap F$ contains points and isolated Baer subplanes. One of these Baer subplanes $\operatorname{PG}(2, \sqrt{q})$ is projected from $R$ onto 
Case III: assume that $F^{\prime}$ contains a Baer subgeometry $\mathrm{PG}(3, \sqrt{q})$.

Consider two Baer subplanes $\omega_{1}$ and $\omega_{2}$ in $\operatorname{PG}(3, \sqrt{q})$. By the arguments of case II, we find Baer subplanes $\omega_{1}^{\prime}$ and $\omega_{2}^{\prime}$ contained in $F$ projected onto $\omega_{1}$ and $\omega_{2}$ respectively. Since there are less than $\frac{q^{1 / 6}}{2}$ multiple points in the intersection line of $\omega_{1}$ and $\omega_{2}$, this projected Baer subline $\omega_{1} \cap \omega_{2}$ must be the projection of a Baer subline contained in $F$, which must be equal to the intersection line of $\omega_{1}^{\prime}$ and $\omega_{2}^{\prime}$. So $\omega_{1}^{\prime}$ and $\omega_{2}^{\prime}$ span a Baer subgeometry $\mathrm{PG}(3, \sqrt{q})$. The 3 -space over $\mathbb{F}_{q}$ defined by this Baer subgeometry shares two intersecting Baer subplanes with $F$. By the induction hypothesis, this Baer subgeometry $\mathrm{PG}(3, \sqrt{q})$ must be contained in $F$.

Conclusion: Let $F$ be a non-weighted $\left\{\epsilon_{1}(q+1)+\epsilon_{0}, \epsilon_{1} ; n, q\right\}$-minihyper, $n \geq 4$, where $\epsilon_{1}+\epsilon_{0}=\eta\left(\sqrt{q}-q^{1 / 6}\right)<\frac{q^{7 / 12}}{2}-\frac{q^{1 / 4}}{2}$.

By assumption, if $F$ contains a line $L$, then this line $L$ can be removed from $F$, so that $F \backslash L$ is an $\left\{\left(\epsilon_{1}-1\right)(q+1)+\epsilon_{0}, \epsilon_{1}-1 ; n, q\right\}$-minihyper (Lemma 2.1). From now on, assume that all the lines contained in $F$ are removed from $F$. By induction on $\epsilon_{1}+\epsilon_{0}$, these lines contained in $F$ are pairwise disjoint among each other.

The preceding cases I, II, and III show that the projection of $F$, from a suitably selected point $R$, onto a hyperplane leads to a minihyper in this hyperplane containing $B^{\prime}$ isolated Baer subplanes $\mathrm{PG}(2, \sqrt{q})$ and $C^{\prime}$ Baer subgeometries $\mathrm{PG}(3, \sqrt{q})$, with $B^{\prime}+C^{\prime}(\sqrt{q}+1)=\epsilon_{1}$, and that these isolated Baer subplanes and 3-dimensional Baer subgeometries contained in the projected minihyper arise from $B^{\prime}$ isolated Baer subplanes $\mathrm{PG}(2, \sqrt{q})$ and $C^{\prime}$ Baer subgeometries $\mathrm{PG}(3, \sqrt{q})$ contained in the minihyper $F$.

If lines again are permitted to be contained in $F$, the preceding arguments therefore show that $F$ is the union of pairwise disjoint $A$ lines, $B$ isolated Baer subplanes $\mathrm{PG}(2, \sqrt{q})$ and $C$ Baer subgeometries $\mathrm{PG}(3, \sqrt{q})$, with $A+$ $B+C(\sqrt{q}+1)=\epsilon_{1}$, and $\epsilon_{0}-B \sqrt{q}$ extra points. 


\section{References}

[1] S. Ball. Multiple blocking sets and arcs in finite planes. J. London Math. Soc. (2), 54(3):581-593, 1996.

[2] J. Barát and L. Storme. Multiple blocking sets in $\mathrm{PG}(n, q), n \geq 3$. Des. Codes Cryptogr., 33(1):5-21, 2004.

[3] A. Blokhuis, L. Storme, and T. Szőnyi. Lacunary polynomials, multiple blocking sets and Baer subplanes. J. London Math. Soc. (2), 60(2):321332, 1999.

[4] J. De Beule, K. Metsch, and L. Storme. Characterization results on arbitrary non-weighted minihypers and on linear codes meeting the Griesmer bound. Des. Codes Cryptogr., 49(1-3):187-197, 2008.

[5] J. De Beule, K. Metsch, and L. Storme. Characterization results on weighted minihypers and on linear codes meeting the Griesmer bound. Adv. Math. Commun., 2(3):261-272, 2008.

[6] S. Ferret and L. Storme. Minihypers and linear codes meeting the Griesmer bound: improvements to results of Hamada, Helleseth and Maekawa. Des. Codes Cryptogr., 25(2):143-162, 2002.

[7] S. Ferret and L. Storme. A classification result on weighted $\left\{\delta v_{\mu+1}, \delta v_{\mu} ; N, p^{3}\right\}$-minihypers. Discrete Appl. Math., 154(2):277-293, 2006.

[8] S. Ferret, L. Storme, P. Sziklai, and Zs. Weiner. A $t(\bmod p)$ result on weighted multiple $(n-k)$-blocking sets in $\mathrm{PG}(n, q)$. Innov. Incidence Geom., 6/7:169-188, 2007/08.

[9] S. Ferret, L. Storme, P. Sziklai, and Zs. Weiner. A characterization of multiple $(n-k)$-blocking sets in projective spaces of square order. Adv. Geom., submitted.

[10] N. Hamada and T. Helleseth. A characterization of some $q$-ary codes $\left(q>(h-1)^{2}, h \geq 3\right)$ meeting the Griesmer bound. Math. Japon., 38(5):925-939, 1993. 
[11] N. Hamada and T. Maekawa. A characterization of some $q$-ary linear codes $\left(q>(h-1)^{2}, h \geq 3\right)$ meeting the Griesmer bound. II. Math. Japon., 46(2):241-252, 1997.

[12] N. Hamada and F. Tamari. On a geometrical method of construction of maximal $t$-linearly independent sets. J. Combin. Theory Ser. A, 25(1):14-28, 1978.

Address of the authors:

Ghent University, Dept. of Mathematics, Krijgslaan 281-S22, 9000 Ghent, Belgium

A. Hallez: athallez@cage.ugent.be

J. De Beule: jdebeule@cage.ugent.be,http://cage.ugent.be/ jdebeule

L. Storme: ls@cage.ugent.be, http://cage.ugent.be/ ls 